\title{
SISTEMÁTICA PARA RACIONALIZAÇÃO DE INSTRUMENTAIS DE BANDEJAS CIRÚRGICAS
}

\author{
A systematic approach for the rationalization of surgical tray instruments \\ Sistemática para racionalización de instrumentales de bandejas quirúrgicas
}

Daniela Silva dos Santos Schneider ${ }^{1 *}$, Michel José Anzanello², Rosane Silva Veiga Pirovano ${ }^{3}$, Flávio Sanson Fogliatto ${ }^{4}$

RESUMO: Objetivo: Relatar a experiência de desenvolver uma sistemática para racionalização de instrumentais em bandejas cirúrgicas. Método: Estudo de desenvolvimento de sistemática para racionalização de instrumentais, realizado em 2015, a partir do método qualitativo, em um centro de materiais e esterilização (CME) de um hospital universitário federal de Porto Alegre, Brasil. Resultados: Houve redução média do quantitativo de instrumentais em bandejas institucionais em 10,92\%; diminuição de bandejas de propriedade das equipes médicas, sendo 84,06\% pertencentes à equipe da otorrinolaringologia; e inativação definitiva de 369 instrumentais da cirurgia ortopédica, o que significou 72,84\% do total dos instrumentais inativados. Além disso, houve condução de melhorias no gerenciamento de instrumentais, otimização do tempo de preparo e redução da esterilização por expiração do prazo de utilização. Conclusão: A realocação de instrumentais e o acréscimo de peças em bandejas específicas permitiu a reavaliação das solicitações de compras de instrumentais e a melhoria das relações entre as equipes. Essa sistemática contribuiu significativamente para o gerenciamento de instrumentais, otimizando processos e envolvendo as equipes cirúrgicas no trabalho do CME e evidenciou que pode ser aplicada em outras instituições.

Palavras-chave: Instrumentos cirúrgicos. Organização e administração. Indicadores de qualidade em assistência à saúde.

ABSTRACT: Objective: To report the experience of developing a systematic approach for the rationalization of instruments in surgical trays. Method: Study of the development of a systematic approach for the rationalization of instruments, carried out in 2015, using a qualitative method, in the Central Sterile Supply Department (CSSD) of a federal university hospital in Porto Alegre, Brazil. Results: There was a 10.92\% average reduction in the number of instruments in institutional trays, a reduction in the number of trays owned by medical teams $-84.06 \%$ belonged to the otorhinolaryngology team - and a definitive inactivation of 369 orthopedic surgery instruments, which represented $72.84 \%$ of the total number of inactivated instruments. In addition, improvements were made to the management of instruments, the optimization of preparation time and the reduction of sterilization by expiration date. Conclusion: The relocation of instruments and the addition of items in specific trays allowed for the reappraisal of requests for purchase of instruments and the improvement of relationships between the teams. This systematic approach contributed significantly to the management of instruments, the optimizing processes and the involvement of the surgical teams in the work of the CSSD, thus demonstrating that it can be applied in other institutions. Keywords: Surgical instruments. Organization and administration. Quality indicators in health care.

RESUMEN: Objetivo: Relatar la experiencia de desarrollar una sistemática para racionalización de instrumentales en bandejas quirúrgicas. Método: Estudio de desarrollo de sistemática para racionalización de instrumentales, realizado en 2015, desde el método cualitativo, en un centro de materiales y esterilización (CSSD) de un hospital universitario federal de Porto Alegre, Brasil. Resultados: Hubo reducción media del cuantitativo de instrumentales en bandejas institucionales en el 10,92\%; disminución de bandejas de propiedad de los equipos médicos, siendo el 84,06\% pertenecientes al equipo de la otorrinolaringología; e inactivación definitiva de 369 instrumentales de la cirugía ortopédica, lo que significó el 72,84\% del total de los instrumentales inactivados. Además, hubo conducción de mejoras en el gerenciamiento de instrumentales, optimización del tiempo de preparo y reducción de la

'Enfermeira; mestre em Toxicologia; especialista em Gestão de Operações em Saúde no Serviço de Enfermagem em Centro Cirúrgico e Centro de Material e Esterilização do Hospital de Clínicas de Porto Alegre - Porto Alegre (RS), Brasil.

2Doutor em Engenharia Industrial e de Sistemas; professor do Departamento de Engenharia Industrial na Universidade Federal do Rio Grande do Sul (UFGRS) - Porto Alegre (RS), Brasil.

${ }^{3}$ Enfermeira; especialista em Gestão de Operações em Saúde e Serviço de Enfermagem em Centro Cirúrgico, na Unidade de Bloco Cirúrgico, do Hospital de Clínicas de Porto Alegre - Porto Alegre (RS), Brasil. ${ }^{4}$ Doutor em Engenharia Industrial e de Sistemas; professor do Departamento de Engenharia Industrial na UFRGS - Porto Alegre (RS), Brasil.

*Autor correspondente: danielassschneider@gmail.com

Recebido: 26/08/2017 - Aprovado: 10/12/2017

DOI: $10.5327 / Z 1414-4425201800010009$ 
esterilización por expiración del plazo de utilización. Conclusión: La reubicación de instrumentales y el incremento de piezas en bandejas específicas permitió la reevaluación de las solicitaciones de compras de instrumentales y la mejora de las relaciones entre los equipos. Esa sistemática contribuyó significativamente para el gerenciamiento de instrumentales, perfeccionando procesos e involucrando a los equipos quirúrgicos en el trabajo de CSSD y evidenció que puede aplicarse en otras instituciones.

Palabras clave: Instrumentos quirúrgicos. Organización y administración. Indicadores de calidad de la atención de salud.

\section{INTRODUÇÃO}

A cada década aumenta o desafio das instituições hospitalares em melhorar a qualidade dos serviços oferecidos à sociedade, principalmente na realização de procedimentos cirúrgicos ${ }^{1,2}$. A qualidade dos processos de limpeza, desinfecção e esterilização está diretamente relacionada à segurança do paciente $\mathrm{e}$ à minimização dos custos com infecções ${ }^{3}$. Nesse contexto, $\mathrm{o}$ centro de material e esterilização (CME) é responsável pelo processamento de produtos para saúde (PPS), englobando ações que iniciam imediatamente após a assistência direta ao paciente ${ }^{4}$.

Além da garantia do processamento dos PPS, no CME deve ocorrer o gerenciamento do inventário de instrumentais, incluindo processos referentes ao registro, acompanhamento, manuseio e gestão do armazenamento de materiais ${ }^{5,6}$. Tais ações buscam reduzir custos operacionais, oferecer quantitativo adequado para o procedimento cirúrgico e segurança no processamento, na funcionalidade e na durabilidade dos materiais 5 .

Devido à grande demanda de materiais processados pelo CME, percebe-se a necessidade da revisão sistemática e periódica do quantitativo de instrumentais utilizados em procedimentos cirúrgicos, como forma de auxiliar na melhoria da qualidade do processo, diminuição do tempo de retorno da bandeja para a equipe cirúrgica, redução do peso das bandejas (fator que influencia na etapa de esterilização) e baixa dos custos com esterilização de peças desnecessárias.

Estudo americano realizado na rede Virginia Mason Medical Center (VMMC), na cidade de Seattle, nos Estados Unidos, identificou redução de aproximadamente 2,8 milhões de dólares após a racionalização do quantitativo de instrumentais disponíveis nas bandejas cirúrgicas. A base para tal redução foi a observação de 20 procedimentos cirúrgicos de diferentes especialidades, quando foram identificados instrumentais que retornavam da sala cirúrgica sem uso. Esse estudo comprovou que a racionalização de instrumentais, utilizando princípios da metodologia Lean, melhorou a qualidade e o tempo do processamento a um custo menor, bem como a eficiência de uso da sala cirúrgica ${ }^{7}$. Ações similares, em outras instituições de saúde, demonstraram que o gerenciamento do inventário de instrumentais de diferentes especialidades auxilia na prevenção de esterilização desnecessária de peças, na otimização do processamento com a diminuição do tempo de resposta e na redução considerável do peso das bandejas ${ }^{8}$.

Outros autores utilizaram a sistemática de agrupamentos, com base na frequência de procedimentos cirúrgicos, diante da utilização dos instrumentais para racionalização de bandejas. A aplicação do algoritmo proposto permitiu identificar novas opções de formação de bandejas que possibilitaram a otimização do processo de esterilização. A literatura evidencia que a aplicação de técnicas de clusterização, que consiste em gerar agrupamento de observações (instrumentais, neste caso) com características similares, e de modelagem, permite propor agrupamentos e soluções ótimas para o processo de racionalização ${ }^{10,12}$.

\section{OBJETIVO}

Relatar a experiência do desenvolvimento de uma sistemática para racionalização de instrumentais em bandejas cirúrgicas, buscando reduzir o volume de material não utilizado no procedimento a ser limpo e esterilizado e realocar os instrumentais entre as bandejas.

\section{MÉTODO}

Foi desenvolvido um estudo com método qualitativo de pesquisa $^{13}$, combinando a abordagem de grupos focais ${ }^{14,15}$. Foram utilizadas abordagens de pesquisa exploratória e descritiva, combinadas com estratégias de intervenção para identificar oportunidades e propor alternativas de melhoria no gerenciamento de instrumentais cirúrgicos. 
A sistemática para racionalização realizou-se no CME de um hospital universitário federal na cidade de Porto Alegre, Brasil. Essa unidade dispõe de, aproximadamente, 80 mil instrumentais. Desse quantitativo, 19.476 peças estão alocadas em 993 bandejas pertencentes à instituição, 78 de propriedade dos cirurgiões e 1.350 peças são embaladas individualmente. Esses instrumentais podem ser utilizados por 17 especialidades, que operam em 38 salas cirúrgicas.

A equipe responsável pelo processamento de materiais é composta por 72 profissionais entre auxiliares e técnicos de enfermagem, 9 enfermeiros (sendo 8 assistenciais e 1 coordenador da unidade), além de 1 auxiliar administrativo. O setor trabalha 24 horas diárias, nos 7 dias da semana. O estudo faz parte de um projeto de desenvolvimento de acompanhamento e avaliação dos processos de gestão em saúde da instituição. O mesmo foi desenvolvido após análise e aprovação do Comitê de Ética do Hospital de Clínicas de Porto Alegre, sob parecer consubstanciado de número 33705014800005327/2014.

O método proposto e utilizado para racionalização de bandejas cirúrgicas foi de desenvolvimento e está apoiado em sete etapas:

- definição de prioridades: a revisão das bandejas priorizou as especialidades cujos procedimentos eram caracterizados por maior complexidade. Na sequência, foram priorizadas as especialidades com maior produção cirúrgica e aquelas sem possibilidade de rodízio dos instrumentais. Por fim, foram revisadas as especialidades que apresentavam o maior quantitativo de ocorrências, no que diz respeito à insatisfação da equipe cirúrgica, bem como sua disponibilidade para realizar a revisão;

- contato com especialista e instrumentador: a partir da definição da especialidade, foi realizada entrevista informal com especialistas do bloco cirúrgico (BC), para agendamento de data e horário para condução da análise dos materiais existentes em cada bandeja, seleção dos instrumentadores que auxiliariam no processo e contato com o cirurgião-chefe da especialidade;

- revisão de bandejas e seleção de instrumentais: a revisão de instrumentais e bandejas foi realizada no CME, com acompanhamento de um enfermeiro e um técnico de enfermagem, pertencentes ao setor, e também dos profissionais da especialidade. Inicialmente, foi avaliada a necessidade de instrumentais embalados individualmente (avulsos) e, posteriormente, a abertura de novas bandejas. A ordem para a revisão das bandejas priorizou aquelas denominadas básicas, utilizadas em todos os procedimentos da especialidade. Os instrumentais foram agrupados segundo sua utilização no plano cirúrgico, objetivando corresponder à sequência de uso dos mesmos na cirurgia. Em seguida, os profissionais da especialidade foram questionados quanto à necessidade de cada material e seu respectivo quantitativo. Repetiu-se o processo para as bandejas especiais. Para essas, o chefe da equipe e o instrumentador foram questionados sobre a necessidade de aumento de bandejas idênticas com base na demanda do agendamento cirúrgico. Nos casos em que houve necessidade desse acréscimo, foi montada nova unidade utilizando os instrumentais que sobraram das bandejas já revisadas;

- documentação das alterações: paralelamente à etapa 3, gerou-se uma planilha (exemplo hipotético no Quadro 1) com vistas ao registro das alterações quantitativas e qualitativas de instrumentais, além do registro de referência do instrumental, tamanho, código da instituição e fotografia dos itens retirados e dos que permaneceram nas bandejas. Tal planilha permitiu, ainda, o rastreamento de materiais alocados em novas bandejas, bem como seu quantitativo. O registro apresentado no Quadro 1 era, então, encaminhado, até 24 horas após a revisão, para as lideranças do CME e dos BC para informação, acompanhamento das alterações e apontamento de eventuais inconsistências na composição das bandejas revisadas;

- revisão dos kits cirúrgicos: após a etapa 2, foi realizada a revisão dos kits cirúrgicos, os quais são compostos pelos materiais que devem ser encaminhados pelo CME para cada procedimento, de acordo com a escala de cirurgias pré-agendadas, ou seja, além das bandejas, foram revisados os instrumentais avulsos, que devem acompanhá-las para o procedimento. A revisão dos kits cirúrgicos de cada especialidade priorizou os procedimentos de menor complexidade, em função da menor quantidade de instrumentais verificados nesse agrupamento. Os profissionais da especialidade foram questionados quanto à necessidade e especificação de bandejas e instrumentais avulsos, além do quantitativo de têxteis a serem encaminhados para cada procedimento cirúrgico. A partir da lista das cirurgias aptas para agendamento cirúrgico no sistema informatizado da instituição, foram realizados agrupamentos das que utilizavam as bandejas e os materiais em quantitativos idênticos e a atualização dos documentos; 
- validação pela equipe cirúrgica: imediatamente no turno ou dia após a revisão, o kit cirúrgico atualizado (com instrumentais e bandejas revisadas) foi encaminhado para realização de procedimento no BC. No momento, ou imediatamente após a cirurgia, foi feita a entrevista com instrumentadores, cirurgiões e lideranças do BC, para avaliação e validação das alterações realizadas. A partir do retorno das informações pelos profissionais, eventuais correções e/ ou revisões foram efetuadas;

- organização do estoque: posteriormente, por meio da ferramenta de gestão visual, foi reorganizado o arsenal/estoque do CME. Para tanto, efetuou-se a identificação das estantes de armazenamento das bandejas, de modo que ficassem agrupadas segundo a especialidade. Para diferenciação das especialidades com bandejas similares foram utilizadas cores definidas, a partir das cores dos prontuários preexistentes das especialidades.

Para o posicionamento das bandejas no estoque, levouse, ainda, em consideração o quantitativo e o peso das bandejas, de modo que itens mais pesados ficassem em posições que facilitassem seu manuseio.

\section{RESULTADOS}

A sistemática proposta foi aplicada nas 11 especialidades cirúrgicas, apresentadas no Quadro 2, contemplando instrumentais pertencentes à instituição e aos cirurgiões. A primeira coluna do quadro apresenta a ordem em que o procedimento de revisão foi executado com relação às especialidades.

Quadro 2. Especialidades cirúrgicas revisadas.

\begin{tabular}{|l|c|}
\hline $\begin{array}{l}\text { Ordem da prioridade da } \\
\text { racionalização }\end{array}$ & \multicolumn{1}{c|}{ Especialidade } \\
\hline 1 & Neurocirurgia \\
\hline 2 & Cirurgia cardiovascular \\
\hline 3 & Cirurgia torácica \\
\hline 4 & Cirurgia vascular \\
\hline 5 & Cirurgia urológica \\
\hline 7 & Cirurgia ortopédica \\
\hline 8 & Cirurgia otorrinolaringológica \\
\hline 9 & Cirurgia plástica \\
\hline 10 & Cirurgia proctológica \\
\hline 11 & Cirurgia pediátrica \\
\hline
\end{tabular}

Quadro 1. Planilha de registro e controle de instrumentais alterados da bandeja da especialidade otorrinolaringologia.

\begin{tabular}{|c|c|c|c|c|c|c|c|}
\hline \multirow{2}{*}{$\begin{array}{l}\text { Otorrinolaringologia } \\
\text { Instrumental }\end{array}$} & \multicolumn{7}{|c|}{$\begin{array}{c}\text { Bandeja de sinusotomia c/ septoplastia } 1 \\
\text { Peças: } 30\end{array}$} \\
\hline & Tamanho & Ref. & $\begin{array}{l}\text { Código } \\
\text { HC }\end{array}$ & \begin{tabular}{|c|} 
Quant. \\
até $16 / 04$
\end{tabular} & $\begin{array}{l}\text { Retirado/ } \\
\text { inserido }\end{array}$ & $\begin{array}{l}\text { Quant. } \\
\text { atual }\end{array}$ & Destino/origem \\
\hline Aspirador curvo & $13 \mathrm{~cm}$ & STORZ 586030 & & 1 & & 1 & \\
\hline $\begin{array}{l}\text { Aspirador Franck-Pasquini } \\
20,5 \mathrm{~cm}\end{array}$ & $2,5 \mathrm{FR}$ & STORZ 662825 & 285915 & 0 & 1 & 1 & peça nova \\
\hline Aspirador grosso & $11 \mathrm{FR} 19 \mathrm{~cm}$ & 701102 & & 1 & & 1 & origem: sinusotomia teste \\
\hline Cubas redondas & $10 \mathrm{~cm}$ & & & 2 & 2 & & desativada \\
\hline Cubas redondas & $8 \mathrm{~cm}$ & & & 2 & 2 & & desativada \\
\hline Cabo de bisturi & No 3 & & & 1 & 1 & & desativada \\
\hline Descolador duplo Cottle & $22,5 \mathrm{~cm}$ & STORZ 479200 & & 0 & 1 & 1 & origem: sinusotomia teste \\
\hline Descolador freer & & 330020 & & 1 & & 1 & \\
\hline Dilatador frontal Ritter no 1 & $14,5 \mathrm{~cm}$ & STORZ 641525 & 216151 & 1 & & 1 & \\
\hline Elevador duplo & $22,5 \mathrm{~cm}$ & STORZ 479000 & 215970 & 1 & & 1 & \\
\hline $\begin{array}{l}\text { Especulo nasal Beckmann } \\
\text { (curvo) }\end{array}$ & $15 \mathrm{~cm}$ & STORZ 400520 & 282205 & 0 & 1 & 1 & peça nova \\
\hline Espéculo nasal grande $55 \mathrm{~mm}$ & $13,5 \mathrm{~cm}$ & STORZ 403565 & 285937 & 0 & 1 & 1 & peça nova \\
\hline Espéculo nasal médio $55 \mathrm{~mm}$ & $13,5 \mathrm{~cm}$ & STORZ 403555 & 285938 & 0 & 1 & 1 & peça nova \\
\hline
\end{tabular}


Em relação aos resultados, verificou-se redução média de $10,92 \%$ (Tabela 1) no número de peças pertencentes à instituição. Essa diminuição foi mais representativa em seis especialidades, destacadas na Tabela 1. Das peças retiradas, 841 instrumentais foram realocados ao almoxarifado do CME, representando retorno ao estoque de $64,10 \%$ para remanejo ou confecção de futuras bandejas.

Outras 498 peças foram desativadas definitivamente. Essa ação se justifica devido às mudanças de técnicas cirúrgicas ou perda definitiva da integridade das peças (Tabela 1). Tais itens serão encaminhados para descarte específico, conforme legislação brasileira.

Percebe-se que, para as especialidades de cirúrgica bucomaxilofacial e vascular, houve aumento do quantitativo de 8,17 e $12,00 \%$ no total de instrumentais constantes nas bandejas (Tabela 1). Esse fato se deve às solicitações das equipes, as quais demandaram adição de peças nas bandejas, bem como aumento do quantitativo de bandejas idênticas. Houve, ainda, nestas especialidades, inativação de outras peças, pouco utilizadas.

No que diz respeito às bandejas de propriedade dos médicos, elas representavam, antes da racionalização, $26,86 \%$ do total de peças do arsenal da CME. Após esse processo, houve uma redução de aproximadamente $10,00 \%$ nesse quantitativo. Nesse sentido, destacam-se os resultados obtidos para a especialidade otorrinolaringologia, para a qual foi verificada redução de $84,06 \%$ da entrega e circulação de instrumentais pertencentes às equipes médicas (Tabela 2).

Acrescenta-se que a racionalização também permitiu detectar instrumentais médicos sem utilização, os quais permaneciam no arsenal e demandavam nova esterilização devido ao vencimento do prazo de validade. Evidencia-se, nessa inativação, a especialidade de cirurgia ortopédica, em que se verificou a ociosidade definitiva de 369 instrumentais, o que corresponde a $72,84 \%$ do total dos instrumentais não utilizados (Tabela 2).

A partir da racionalização foram, ainda, reavaliados os pedidos de compras de novas bandejas. Desses, destaca-se a especialidade urológica, na qual havia necessidade de aquisição de 38 instrumentais para confecção de nova bandeja para atender à demanda de cirurgias. Contudo, a partir da revisão de outras três especialidades (neurologia, torácica e cardíaca) e adição de cinco instrumentais disponíveis no almoxarifado do CME, foi possível disponibilizar à equipe de urologia uma nova bandeja com instrumentais idênticos àqueles com pedido de compra. Tal ação respondeu por uma economia de, aproximadamente, $\mathrm{R} \$ 14.000,00$ na compra desses instrumentos. Situação similar ocorreu nas demais dez especialidades, que tiveram suas listas de pedido de compras individuais revisadas.

Tabela 1. Resultados da racionalização de instrumentais e bandejas institucionais.

\begin{tabular}{|c|c|c|c|c|c|c|c|c|c|}
\hline \multirow{3}{*}{ Especialidade } & \multicolumn{3}{|c|}{ Racionalização de bandejas } & \multicolumn{6}{|c|}{ Racionalização de peças } \\
\hline & Antes & Após & Redução & Antes & Após & Redução & Desativadas & Realocadas & Adicionadas \\
\hline & $\mathbf{n}$ & $\mathbf{n}$ & $\%$ & $\mathbf{n}$ & $\mathbf{n}$ & $\%$ & $\mathbf{n}$ & $\mathbf{n}$ & $n$ \\
\hline Neurocirurgia & 58 & 44 & $-24,14$ & 812 & 648 & $-20,20$ & 2 & 141 & 3 \\
\hline Cirurgia cardiovascular & 32 & 31 & $-3,13$ & 862 & 816 & $-5,34$ & 20 & 48 & 48 \\
\hline Cirurgia torácica & 21 & 18 & $-14,29$ & 769 & 583 & $-24,19$ & 25 & 136 & 22 \\
\hline Cirurgia vascular & 28 & 28 & - & 901 & 1.016 & 12,76 & 1 & 10 & 56 \\
\hline Cirurgia urológica & 34 & 31 & $-8,82$ & 689 & 625 & $-9,29$ & 1 & 75 & 52 \\
\hline Cirurgia ortopédica & 89 & 68 & $-23,60$ & 1.969 & 1.666 & $-15,39$ & 263 & 62 & 65 \\
\hline $\begin{array}{l}\text { Cirurgia } \\
\text { otorrinolaringo-lógica }\end{array}$ & 31 & 26 & $-16,13$ & 1.315 & 1.171 & $-10,95$ & 18 & 198 & 181 \\
\hline Cirurgia plástica & 25 & 23 & $-8,00$ & 1.042 & 849 & $-18,52$ & 129 & 67 & 41 \\
\hline Cirurgia proctológica & 12 & 11 & $-8,33$ & 138 & 113 & $-18,12$ & 18 & - & - \\
\hline Cirurgia pediátrica & 21 & 19 & $-9,52$ & 807 & 752 & $-6,82$ & - & 11 & 32 \\
\hline $\begin{array}{l}\text { Cirurgia } \\
\text { bucomaxilofacial }\end{array}$ & 10 & 10 & - & 257 & 278 & 8,17 & 21 & 93 & 138 \\
\hline Total & 361 & 309 & $-14,40$ & 9.561 & 8517 & $-10,92$ & 498 & 841 & 638 \\
\hline
\end{tabular}


Tabela 2. Resultados da racionalização de instrumentais e bandejas de propriedade do cirurgião.

\begin{tabular}{|c|c|c|c|c|c|c|c|c|c|}
\hline \multirow{3}{*}{ Especialidade } & \multicolumn{3}{|c|}{ Racionalização de bandejas } & \multicolumn{6}{|c|}{ Racionalização de peças } \\
\hline & Antes & Após & Redução & Antes & Após & Redução & Desativadas & Realocadas & Adicionadas \\
\hline & $\mathbf{n}$ & $\mathbf{n}$ & $\%$ & $\mathbf{n}$ & $\mathbf{n}$ & $\%$ & $\mathbf{n}$ & $\mathbf{n}$ & $\mathbf{n}$ \\
\hline Neurocirurgia & 7 & 5 & -29 & 74 & 61 & $-17,57$ & 13 & - & - \\
\hline Cirurgia cardiovascular & 37 & 32 & -14 & 192 & 182 & $-5,21$ & 3 & - & - \\
\hline Cirurgia torácica & 4 & 4 & - & 43 & 42 & $-2,33$ & 1 & - & - \\
\hline Cirurgia vascular & 5 & 5 & - & 13 & 13 & - & - & - & - \\
\hline Cirurgia ortopédica & 26 & 6 & -77 & 473 & 104 & $-78,01$ & 369 & - & - \\
\hline $\begin{array}{l}\text { Cirurgia } \\
\text { otorrinolaringo-lógica }\end{array}$ & 17 & 9 & -47 & 138 & 22 & $-84,06$ & 116 & - & - \\
\hline Total & 96 & 61 & -36 & 933 & 424 & $-54,53$ & 474 & - & - \\
\hline
\end{tabular}

\section{DISCUSSÃO}

Além dos benefícios decorrentes dos indicadores de redução apresentados, a racionalização de instrumentais das bandejas pertencentes a 11 especialidades também resultou na melhoria da relação entre as equipes cirúrgicas e o CME. Esse fato se deve ao compartilhamento das responsabilidades em relação à escolha dos instrumentais que deveriam ficar nas bandejas e ao quantitativo de conjuntos idênticos disponíveis para agendamento dos procedimentos, além da visita ao CME e à explicação de cada etapa e do tempo necessário para processamento dos materiais. Esse conhecimento, por parte dos cirurgiões e instrumentadores, auxiliou no planejamento da quantidade, na ordem de realização de cirurgias e na prioridade do encaminhamento dos materiais ao CME, além de melhorar a confiança no trabalho realizado pelos profissionais desse setor. Esse aspecto foi verbalizado pelo retorno feito pelas equipes.

Em termos financeiros, a racionalização das bandejas pode conduzir a uma redução no número de ciclos da autoclave para (re)esterilização, ou seja, necessidade de (re)processar bandejas que não foram utilizadas e que tiveram o prazo de validade expirado.

Outro fato gerador de otimização é a redução do tempo de preparo das bandejas, aproximadamente $28 \%$ menor (diferença entre o tempo de preparo e de empacotamento de bandejas revisadas), diante da cronoanálise realizada antes da racionalização e publicada em outro estudo da própria instituição ${ }^{16}$. Tais resultados ratificam os achados da literatura ${ }^{7-10,12}$.

Enfatiza-se, também, que a realocação de instrumentais decorrentes do procedimento de racionalização e o acréscimo de outras peças em bandejas específicas permitiu o agendamento de maior número de cirurgias subsequentes. A partir desse procedimento, identificou-se a necessidade de antecipar a etapa de recebimento de instrumentais pelo CME na unidade de BC como forma de auxiliar na conferência dos mesmos, na identificação imediata de manutenção do instrumental, no melhor relacionamento com as equipes cirúrgicas e de enfermagem perioperatória e otimização do tempo de processamento.

No que diz respeito às bandejas de propriedade dos médicos, houve redução na quantidade devido, principalmente, à readequação das bandejas institucionais, as quais passaram a suprir, de forma mais adequada, a demanda dos cirurgiões. De tal forma, verificou-se redução do número de eventos em que instrumentais médicos eram recebidos sem ou com pouco tempo hábil para esterilização.

\section{CONSIDERAÇÕES FINAIS}

Neste estudo, o uso da sistemática desenvolvida para a racionalização de instrumentais em bandejas cirúrgicas auxiliou na melhoria do gerenciamento do CME e na relação com as equipes cirúrgicas. Houve redução do quantitativo de instrumentais, aprimoramento do processo de trabalho e, além disso, trouxe avanços no que diz respeito ao gerenciamento dos pedidos de compras de instrumentais.

Entre os aspectos positivos do estudo, também destacase que o mesmo subsidiou o processo de planejamento de compra de instrumental cirúrgico.

A partir dos resultados obtidos com a racionalização de instrumentais de 11 especialidades, percebe-se que é fundamental estender a racionalização para as demais especialidades cirúrgicas e com instrumentais nas unidades de internação da instituição. Concluída tal etapa, propõe-se realizar um estudo 
estimando os custos alocados no arsenal do CME, o quantitativo de peças em almoxarifado e o investimento financeiro necessário, projetando as novas instalações institucionais e o decorrente aumento da capacidade de produção cirúrgica.

Esses dados subsidiam a implantação de sistema informatizado de gerenciamento de instrumentais, contemplando a rastreabilidade das etapas do processo, controle de insumos, custos e registros de manutenção dos instrumentais, auxiliando no planejamento de investimentos em aquisição de novos instrumentos. Portanto, a sistemática de racionalização de instrumentais contribui para pesquisa e ensino, no que se refere aos aspectos gerenciais e assistenciais, principalmente porque o estudo impacta diretamente na segurança do paciente que se submete a um procedimento anestésico-cirúrgico.

A utilização exclusiva da opinião dos especialistas pode ser entendida como uma limitação da sistemática proposta neste estudo, bem como a não realização com todas as especialidades cirúrgicas e o fato de ter sido executado em uma instituição de ensino.

Propõem-se futuros estudos com observação de campo e utilização de ferramentas de programação aplicadas na engenharia de produção, nas diversas especialidades e em outras instituições.

Conclui-se que esta sistemática contribuiu significativamente para o gerenciamento de instrumentais, otimizando processos e envolvendo as equipes cirúrgicas ao trabalho do CME. Ficou evidenciado que esse método deve ser ampliado, podendo ser aplicado em outras instituições, pois contribui diretamente para melhoria do processo de trabalho, que repercute positivamente nos cuidados aos pacientes submetidos a cirurgias de diversas especialidades.

\section{REFERÊNCIAS}

1. Graziano KU, Lacerda RA, Turrini RTN, Calicchio LG. Indicadores de qualidade do centro de material e esterilização. In: Graziano KU, Silva A, Pasltikidis EM, eds. Enfermagem em centro de material e esterilização. Barueri: Manole; 2011. p. 284-300. Capítulo 13.

2. Possari JF. Centro cirúrgico: planejamento, organização e gestão. $4^{\mathrm{a}}$ ed. São Paulo: látria; 2010.

3. Associação Paulista de Epidemiologia e Controle de Infecção Relacionada à Assistência à Saúde (APECIH). Limpeza, desinfecção e esterilização de artigos em serviços de saúde. São Paulo: APECIH; 2010.

4. Brasil. Ministério da Saúde (MS). Agencia Nacional de Vigilância Sanitária (ANVISA). Resolução de Diretoria Colegiada RDC n. 15, de 15 de março de 2012. Dispõe sobre requisitos de boas práticas para o processamento de produtos para saúde e dá outras providências. Brasília (DF): Ministério da Saúde; 2012.

5. Bronzatti JAG, Turrini RNTT, Moriya GAA. Controle de instrumental no centro de material e esterilização. In: Graziano KU, Silva A, Psaltikidis EM, eds. Enfermagem em centro de material e esterilização. São Paulo: Manole; 2011. p. 263-84.

6. Associação Brasileira de Enfermeiros de Centro Cirúrgico, Recuperação Anestésica e Centro de Material e Esterilização (SOBECC). Diretrizes de práticas em enfermagem cirúrgica e processamento de produtos para a saúde. 7ª ed. Baruei: Manole; São Paulo: SOBECC; 2017.

7. Farrokhi RF, Gunther M, Williams B, Blackmore CC. Application of lean methodology for improved quality and efficiency in operating room instrument availability. J Healthc Qual. 2015;37(5):277-86. DOI: 10.1111/jhq.12053
8. Greenberg JA, Wylie B, Robinson JN. A pilot study to assess the adequacy of the Brigham 20 Kit for cesarean delivery. Int J Gynaecol Obstet. 2012;117(2):157-9. DOl: 10.1016/j.ijgo.2011.12.007

9. Reymondon F, Pellet B, Marcon E. Optimization of hospital sterilization costs proposing new grouping choices of medical devices into packages. Int J Prod Econ. 2008;112(1):326-35.

10. Cardoen B, Beliën J, Vanhoucke M. On the design of custom packs: grouping of medical disposable items for surgeries. Int J Prod Res. 2015;53(24):7343-59. https://doi.org/10.1080/00207543.2015.1061221

11. Agard B, Penz B. A simulated annealing method based on a clustering approach to determine bills of materials for a large product family. Int J Prod Econ. 2009;117(2):389-401. DOI: 10.1016/j.ijpe.2008.12.004

12. Dobson G, Froix A, Seidmann A, Tilson V, Froix A. Configuring surgical instrument trays to reduce costs. IISE Trans Health Syst Eng. 2015;5(4):225-37. https://doi.org/10.1080/19488300.2015.1094759

13. Keptner KM. Mixed methods design: a beginner's guide. The Qualitative Report. 2011;16(2):593-95.

14. Ressel LB, Beck CLC, Gualda DMR, Hoffmann IC, Silva RMD, Sehnem GD. 0 uso do grupo focal em pesquisa qualitativa. Texto \& Contexto Enferm. 2008;17(4):779-86. http://dx.doi.org/10.1590/S0104-07072008000400021

15. Barbour R. Grupos Focais. Porto Alegre: Artmed; 2009. 216p.

16. Fogliatto FS, Anzanello MJ, Tortorella GL, Schneider DS, Pereira CG, Schaan BD. A Six Sigma approach to analyze time-to-assembly variance of surgical trays in a sterile services department. J Healthc Qual. 2017 Mar 24. DOI: 10.1097/JHQ.0000000000000078 